\title{
Large benign retroperitoneal tumour in pregnancy
}

\author{
Csaba Berczi, MD, PhD; Peter Osvath, MD; Tibor Flasko, MD, PhD
}

Department of Urology, University of Debrecen, Debrecen, Hungary

Cite as: Can Urol Assoc J 2015;9(7-8):E551-3. http://dx.doi.org/10.5489/cuaj.2908

Published online August 10, 2015.

\section{Abstract}

A 31-year-old female was in the 13th week of pregnancy when an abdominal ultrasound examination revealed a large retroperitoneal tumour. Magnetic resonance imaging was carried out and the imaging described a $10-\mathrm{cm}$ mass in diameter extending from the right kidney. Given that the patient was in her first trimester and that there was a suspicion of malignancy, further surgical exploration of the tumour was warranted. During the operation, the tumour was removed, but nephrectomy was not necessary. Histologic analysis of the resected tumour showed a mucinous cystic adenoma, and no signs of malignancy were present. Following the surgery, the pregnancy was otherwise uneventful and further complications did not occur. This case illustrates that surgery is recommended in patients with a retroperitoneal tumour early during a pregnancy, when a malignancy cannot be excluded.

\section{Introduction}

Retroperitoneal tumours, which develop in the retroperitoneum without originating from the major retroperitoneal organs, are quite rare. Retroperitoneal tumours can be defined as cystic or solid masses based on the radiomorphology. In regards to their biological behaviour, they can be divided into benign or malignant tumours. Most malignancies are generally primary tumours, but metastatic lesions may also occur in this region. ${ }^{1}$

About $70 \%$ to $80 \%$ of the primary retroperitoneal neoplasms are malignant. ${ }^{1}$ The most common malignant tumours in this region are sarcomas. Apart from sarcomas, lymphomas, epithelial malignancies, and metastases of different germ cell tumours may present in the retroperitoneum. The most common benign lesions in the retroperitoneum are lipomas, fibromas, and benign neurogenic tumours.

During pregnancy, both benign and malignant retroperitoneal tumours are extremely rare.

\section{Case report}

A 31-year-old primigravid patient in the 13th week of gestation came to our department because of pain in the right lumbocostal area and a 2-week fever.

The ultrasound described a 10-cm mass near the upper pole of the right kidney. This lesion was mostly solid with cystic components, making an impression on the lower surface of the liver. Since the details expressed the possibility of a malignancy, it was decided to perform an urgent magnetic resonance imaging (MRI). A native MRI was performed since the patient had had a previous hypersensitivity to a contrast agent. The MRI described a 10-cm tumour in the retroperitoneum above the right kidney, which was capable of dislocating and compressing the kidney, the liver, and the inferior vena cava. The tumour was well-circumscribed, with partly solid and fluent portions separated by septums (Fig. 1, Fig. 2).

Since the patient was in her first trimester and there was a possibility of malignancy, it was decided to perform a surgical extraction of the tumour. In preparation for the operation, consultations were carried out with a genetic consultant. The genetic consultant felt that the operation posed no increased fetal risk over the normal of $3 \%$.

The retroperitoneum was explored via an oblique lumbar incision. A large tumour was found above the right kidney tightly fused with the peritoneum, the diaphragm, and its environment, while pressing upon the inferior vena cava. During the operation, the whole tumour was removed but nephrectomy was not needed. There were no postoperative complications. The histologic result showed mucinous benign cystic adenoma. There were no histological signs of malignancy.

Afterwards, the pregnancy was uneventful and an abortion was not necessary. On the 39th week of gestation, a healthy child was born. At the 2-year follow-up, there were no further complications in the patient or her child. 


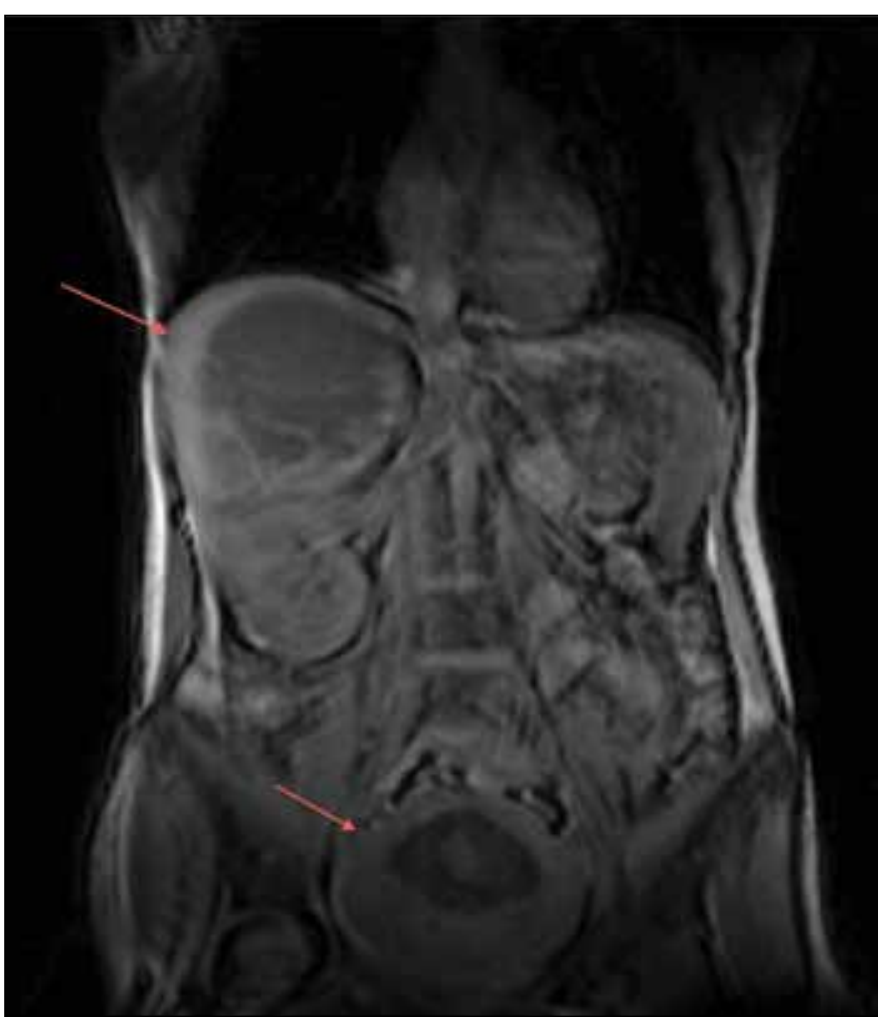

Fig. 1. Retroperitoneal mass in a pregnant patient. Coronal magnetic resonance image shows the retroperitoneal tumour and the embryo in the uterus.

\section{Discussion}

Primary retroperitoneal tumours occur in less than $0.2 \%$ of all malignancies. Once the retroperitoneal masses have been removed, only a few cases are benign by histology.

The most frequent tumours are the soft tissue sarcomas, which comprise about a third of retroperitoneal tumours. ${ }^{1}$ Other common retroperitoneal malignancies may be primary lymphoproliferative tumours and malignant histiocytomas. Metastatic tumours may also present in this region, and when they do, they primarily result from metastases of germ cell tumours. Benign retroperitoneal tumours are extremely rare. The most common benign lesions in the retroperitoneum are lipomas, benign neurogenic tumours, paragangliomas and fibromatosis.

In the patient's case, the histology showed that the mass was a cystadenoma, which is a very rare tumour in the retroperitoneum. Less than 50 cases of primary retroperitoneal mucinous cystadenomas have been reported. ${ }^{2}$ The occurrence of this tumour type is unusual and its pathogenesis is not completely understood since epithelial cells are not normally present in the retroperitoneum for adenomas and adenosarcomas to develop. ${ }^{2,3}$

Retroperitoneal tumours are extremely rare in pregnancy, with only a few reported cases of primary tumours. ${ }^{3-6}$ To the best of our knowledge, retroperitoneal cystadenoma during

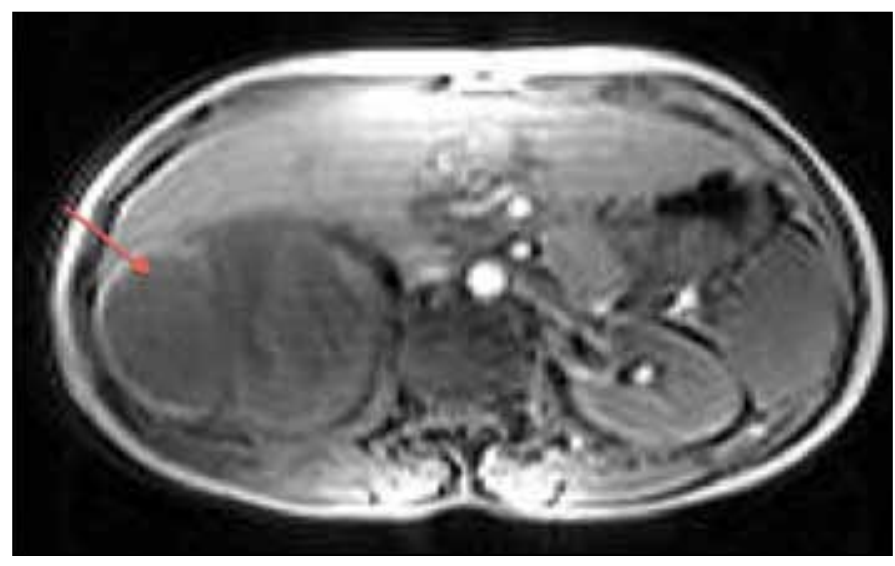

Fig. 2. Axial T1-weighted magnetic resonance image shows the mass in the right retroperitoneum.

pregnancy has not been reported. The lack of experience poses both a diagnostic problem and a therapeutic challenge.

Generally, retroperitoneal tumours are asymptomatic until they have reached a substantial size. For this reason, they are often diagnosed by a routine ultrasound as an incidental finding during an investigation for nonspecific complaints. Contrast-enhanced computed tomography (CT) and MRI are important in both the diagnosis and differential diagnosis of retroperitoneal tumours.

Since the differential diagnosis between malignant and benign masses in retroperitoneum may not be resolved by imaging, it is safer for patients with a suspicion of malignancy to be treated by surgery. Complete tumour resection is recommended since the extent of the operation will determine the prognosis in malignant lesions. ${ }^{1,4}$

During pregnancy, surgery may be performed by an open surgical intervention or by laparoscopic technique. In these cases, however, it must be remembered that, instrumental manipulation during the laparoscopy is more difficult due to the growing uterus. In addition to this hurdle, the increased intra-abdominal pressure may reduce the placental circulation, the cardiac output, and maternal venous return during the laparoscopy.

Tariel and colleagues reported their experience with the management of ovarian tumours in pregnancy. It was reported that the risk of miscarriage following surgery both through laparotomy and laparoscopy for ovarian tumours is between 3\% and 5\%. Laparoscopic surgery is possible in the first and second trimester of pregnancy for the management of presumed benign ovarian tumours. ${ }^{7}$

The timing of the operation mainly depends on the gestational age. If the tumour is discovered in the first trimester, then the surgery should not be postponed. When it is diagnosed in the second trimester, we can try to delay the operation until week 28, when the lungs of the foetus would have reached adequate maturity, but it depends on 
the individual clinical situation. Surgeries performed in the second trimester are predisposed to uterine contractions, which may result in fetal distress or even pre-term birth. In cases where the tumour is diagnosed in the third trimester, the operation should be performed in combination with a Caesarean section. Most surgeries in these cases have been performed postpartum or at the time of Caesarean section. ${ }^{8}$ However, it must be remembered that delaying surgery has a potential risk to the patient since distant metastasis and a local tumour expansion may occur.

In our patient's case, certain diagnostic difficulties were present. A CT was not taken to avoid the possible radiation injury of the embryo. While MRI was possible, its usefulness in this case was limited since the patient's sensitivity to contrast media allowed only native MRI to be taken. At presentation, the tumour was large and contained heterogenous structures. For this reason, fine needle biopsy was not performed since the biopsy specimen would not be able to exclude the presence of malignancy. These diagnostic dilemmas increased the risk of watchful waiting.

Given that the tumour presented in the 13th week of gestation, a delayed operation could have had serious patient implications. In the 14th week of the pregnancy, a complete surgical resection of the retroperitoneal tumour was performed. In the end, the histology of the removed retroperitoneal tumour revealed a benign lesion.

\section{Conclusion}

During pregnancy, both benign and malignant retroperitoneal tumours are extremely rare. When retroperitoneal tumours are discovered early during pregnancy, it is our recommendation to do a complete resection, particularly when malignancy cannot be excluded.

Competing interests: The authors all declare no competing financial or personal interests.

This paper has been peer-reviewed.

\section{References}

1. Strauss DC, Hayes AJ, Thomas JM. Retroperitoneal tumours: Review of management. Ann R Coll Surg Engl 2011;93:275-80. http://dx.doi.org/10.1308/003588411X571944

2. Bifulco $G$, Mandato VD, Giampaolino P, et al. Huge primary retroperitoneal mucinous cystadenoma of borderline malignancy mimicking an ovarian mass: Case report and review. Anticancer Res 2008;28:230915.

3. Kashima K, Yahata T, Fujita K, et al. Primary retroperitoneal mucinous cystadenocarcinoma associated with pregnancy. Int J Gynecol Cancer 2008;18:908-12. http://dx.doi.org/10.1111/i.15251438.2007.01130.x

4. Ulker V, Gungorduk K, Numanoglu C, et al. Complete surgical resection of retroperitoneal leiomyosarcoma in pregnancy: A case report. Arch Gynecol Obstet 2008;277:353-6. http://dx.doi.org/10.1007/ s00404-007-0457-7

5. Due-as-García OF, Díaz-Sotomayor M, Rico-Olvera H. Well differentiated giant retroperitoneal liposarcoma during the pregnancy. Rev Esp Enferm Dig 2011;103:657-8. http://dx.doi.org/10.4321/S113001082011001200012

6. Lopes RI, Machado M, Paz C, et al. Successful outcome of a surgically treated giant retroperitoneal liposarcoma during pregnancy. Arch Gynecol Obstet 2009;280:1067-9. http://dx.doi.org/10.1007/ s00404-009-1061-9

7. Tariel 0 , Huissoud C, Rudigoz RC, et al. Presumed benign ovarian tumors during pregnancy. J Gynecol Obstet Biol Reprod (Paris) 2013;42:842-55. http://dx.doi.org/10.1016/i.gyn.2013.09.038

8. Huo D, Liu L, Tang Y. Giant retroperitoneal liposarcoma during pregnancy: A case report. World I Surg Oncol 2015;13:145. http://dx.doi.org/10.1186/s12957-015-0555-0

Correspondence: Dr. Csaba Berczi, Department of Urology, University of Debrecen, Nagyerdei str. 98, Debrecen, Hungary, H-4032; berczi@med.unideb.hu 